\title{
Flexible high-energy-density lithium-sulfur batteries using nanocarbon-embedded fibrous sulfur cathodes and membrane separators
}

\author{
Jun-Woo Park', Seong-Chan Jo, ${ }^{1,2}$, Min-Ju Kim¹, Ik-Hyeon Choi', Byung Gon Kim¹, You-Jin Lee', Hae-Young Choi', \\ Sung Kang ${ }^{3}$, TaeYoung Kim $\mathbb{1}^{4}$ and Kang-Jun Baeg $\mathbb{1 0}^{2,5}$
}

\begin{abstract}
To obtain soft electronics, it is essential to develop high-performance and mechanically flexible energy storage at the industry level. Herein, we report flexible high-energy-density lithium-sulfur (Li-S) batteries based on all-fibrous sulfur cathodes and separators. To implement free-standing and flexible sulfur cathodes, electrically conductive single-walled carbon nanotubes (CNTs) are impregnated with cellulose nanofibers. This fibrous structure forms a 3D porous electrode with a large surface area to improve redox kinetics and achieve a high sulfur loading content without the use of a metal collector, which can then be applied in high-energy-density batteries. These flexible sulfur cathodes are combined with a commercial glass fiber separator coated with a CNT layer through a cost-effective solution process to suppress the shuttle effects of lithium-polysulfide, thereby exhibiting robust cycling stability. The prepared Li-S batteries exhibit high capacities of $940 \mathrm{mAh} \mathrm{g}^{-1}$ at a charge current density of $1.57 \mathrm{~mA} \mathrm{~cm}^{-2}$ and at $25^{\circ} \mathrm{C}$, and the Coulombic efficiency exceeds $90 \%$ even after 50 charge/discharge cycles. Moreover, Li-S batteries with a high gravimetric energy density of $443 \mathrm{Wh} \mathrm{kg}^{-1}$ per cell is achieved, and these batteries demonstrate excellent reliability in regard to electrochemical performance even under severe mechanical stress conditions for over 100 cycles.
\end{abstract}

\section{Introduction}

Recently, some pioneering companies have launched innovative mobile smart devices, such as the Samsung Galaxy Z Flip and Huawei Mate X, which are foldable $5 \mathrm{G}$ smartphones. Although these foldable phones are not mainstream devices, this technological trend will be maintained further and is also expected to be accelerated by the development of new materials and components. Wearable smart devices have also received considerable attention from researchers, stimulating

\footnotetext{
Correspondence: TaeYoung Kim (taeykim@gachon.ac.kr) or Kang-Jun Baeg (kangjun100@pknu.ac.kr)

${ }^{1}$ Next-Generation Battery Research Center, Korea Electrotechnology Research Institute, 12 Bulmosan-ro 10beon-gil, Seongsan-gu, Changwon-si, Gyeongsangnam-do 51543, Republic of Korea

${ }^{2}$ Department of Smart and Green Technology Engineering, Pukyong National University, 45 Yongso-ro, Nam-gu, Busan 48513, Republic of Korea Full list of author information is available at the end of the article These authors contributed equally: Jun-Woo Park, Seong-Chan Jo
}

increasing research for a variety of applications, such as real-time healthcare monitoring, wearable-assistive robotics or exoskeletons, augmented reality, and entertainment for humans ${ }^{1,2}$. Given the increasing demands for flexible and wearable electronics, it is necessary to develop corresponding energy storage devices that are mechanically flexible, namely, that these developed materials are foldable and even stretchable $^{3}$. These emerging energy storage devices also need to be lightweight and have high electrochemical performance with a high energy density, high rate capability, and long cycling life ${ }^{4-7}$. In comparison with remarkable progress in regard to the other critical components in next-generation smart devices, such as flexible printed circuit boards and flexible or foldable displays $^{8,9}$, the development of rechargeable batteries with mechanical flexibility and high performance has been considerably slower.

\section{(c) The Author(s) 2021}

(c) (i) Open Access This article is licensed under a Creative Commons Attribution 4.0 International License, which permits use, sharing, adaptation, distribution and reproduction cc) in any medium or format, as long as you give appropriate credit to the original author(s) and the source, provide a link to the Creative Commons license, and indicate if changes were made. The images or other third party material in this article are included in the article's Creative Commons license, unless indicated otherwise in a credit line to the material. If material is not included in the article's Creative Commons license and your intended use is not permitted by statutory regulation or exceeds the permitted use, you will need to obtain permission directly from the copyright holder. To view a copy of this license, visit http://creativecommons.org/licenses/by/4.0/. 
To date, lithium-ion batteries (LIBs) have been widely used for portable electronic devices and electric vehicles owing to their high energy density $\left(\sim 300 \mathrm{Wh} \mathrm{kg}^{-1}\right)$, high operating voltage, and long cycling lifetime. However, the widespread adoption of LIBs in flexible electronics is limited by their unstable electrochemical functions under mechanical deformation conditions ${ }^{10}$. Among the various battery systems being discussed for next-generation electronics or electrical applications, lithium-sulfur (Li-S) batteries have been considered a promising alternative to LIBs in terms of their low cost, nontoxicity, and natural abundance of available cathode active materials ${ }^{11}$. The theoretical capacity of sulfur cathodes is $\sim 10$ times larger than that of conventional cathode materials used in LIBs, such as lithium nickel cobalt manganese $\left(\mathrm{LiNi}_{1-\mathrm{x}-\mathrm{y}} \mathrm{Co}_{\mathrm{x}} \mathrm{Mn}_{\mathrm{y}} \mathrm{O}_{2}\right.$, layerstructured), nickel cobalt aluminum $\left(\mathrm{LiNi}_{1-\mathrm{x}-\mathrm{y}} \mathrm{Co}_{\mathrm{x}} \mathrm{Al}_{\mathrm{y}} \mathrm{O}_{2}\right.$, layer-structured) and lithium iron phosphate $\left(\mathrm{LiFePO}_{4}\right.$, olivine structured $)^{12}$. The high capacity and charge/discharge cycling performance of these materials results from their two-electron system during the electrochemical reaction. During the discharge process, every sulfur atom can transfer two electrons by breaking the $\mathrm{S}-\mathrm{S}$ bonds in the crown-like $\mathrm{S}_{8}$ structure. Therefore, Li-S batteries can exhibit a specific energy density of $\sim 2500 \mathrm{Wh} \mathrm{kg}^{-1}$, which is five- to tenfold higher than that of LIBs ${ }^{7,13-16}$.

Owing to their many advantageous properties, Li-S batteries have been explored as replacements for conventional LIBs in flexible electronics. Despite their high theoretical specific capacity and energy density, current $\mathrm{Li}-\mathrm{S}$ batteries are still limited by the low electrical and ionic conductivities of elemental sulfur, large volume changes between sulfur and $\mathrm{Li}_{2} \mathrm{~S}$, structural instability with conventional polymeric binders, deterioration of lithium anodes with dendrite growth and surface passivation, and shuttle phenomena of dissolved lithium polysulfides $\left(\mathrm{Li}_{2} \mathrm{~S}_{\mathrm{x}}\right)$ in the electrolyte ${ }^{17}$. In general, a Li-S battery consists of several components, such as a sulfur cathode, binder, separator, electrolyte, anode, and collector. Among them, elemental sulfur and its cathode are the most crucial materials because they are strongly related to the structures of the cells and determine their appreciable electrochemical performance. Typically, a mixture of sulfur and carbon-based conductive additives is deposited on the metallic current collector by a slurry-based casting $\operatorname{method}^{4,18,19}$. However, with this casting process, it is challenging to achieve high energy density because the metallic foil is an inactive component, resulting in a decrease in the gravimetric energy density of batteries ${ }^{20-24}$. Moreover, active materials also easily detach from the metallic current collector under mechanical stress conditions.

Regarding the materials of flexible $\mathrm{Li}-\mathrm{S}$ batteries, carbon-based nanomaterials are the primary choice owing to their excellent electrical and mechanical properties, large surface area, high chemical stability, and unique structural features that can provide efficient pathways for both electrons and lithium ions. They also enable fast redox kinetics and the presence of active sites, leading to high energy and power densities, charge/discharge cycling life, and rate capabilities. In this regard, many research groups have reported developments based on nanostructured carbon-based sulfur cathodes for Li-S batteries, such as the formation of highly ordered interwoven composites $^{6}$, impregnated sulfur in the micropores of activated carbon fibers ${ }^{25}$, a high sulfur-loaded film electrode based on carbon nanotube (CNT)-coated conductive carbon fibers ${ }^{26}$, a flexible all-carbon structure of interlinked nanotubes ${ }^{27}$, an integrated structure of sulfur and a graphene-coated commercial separator ${ }^{5}$, and all fibrous cathode-separator assemblies ${ }^{4}$. These approaches can contain more active materials inside the nanostructured electrodes and alleviate the capacity attenuation problem of Li-S cells during the cycling process by addressing the "shuttle effect" of $\mathrm{Li}_{2} \mathrm{~S}_{\mathrm{x}}$. Despite the fact that tremendous efforts have been devoted to overcoming the problems mentioned above, the exploitation of nanomaterials in flexible $\mathrm{Li}-\mathrm{S}$ batteries remains a formidable task due to limitations on their mechanical flexibility, electrochemical performance, and capacity retention during charge/discharge cycles.

Here, we propose flexible $\mathrm{Li}-\mathrm{S}$ batteries based on the fibrous composition of sulfur cathodes that consist of a cellulose nanofiber (CNF) framework and electronically conductive CNT additives. Instead of slurry casting sulfurcarbon composites on a metallic current collector with a polymeric binder, we apply CNT-impregnated CNFs directly to create a free-standing electrode. Combined with sulfur-encapsulated multiwalled carbon nanotubes (MWCNTs), they form a unique three-dimensional cathode structure without a metallic current collector and a polymeric binder ${ }^{28,29}$. Nanocarbon-based fibrous sulfur cathodes are assembled into a $\mathrm{Li}-\mathrm{S}$ battery with a CNTcoated GA55 commercial separator, in which the cell exhibits a very high energy density $\left(443 \mathrm{Wh} \mathrm{kg}_{\text {cell }}{ }^{-1}\right)$ and stable charge retention characteristics. Since the $\mathrm{Li}-\mathrm{S}$ battery exhibits stable electrochemical performance without a short circuit even when crumbled or rolled, the material design and manufacturing approaches described herein can realize low-cost, flexible, and high-energydensity $\mathrm{Li}-\mathrm{S}$ batteries for future flexible electronic applications.

\section{Materials and methods}

\section{Fabrication of the CNF-based sulfur cathodes and CNT- coated GA55 separator}

Sulfur powder ( $<98 \%$, Wako Chemical, Japan) was mixed with MWCNTs (diameter $=9.5 \mathrm{~nm}$, Nanocyl, Belgium) (Sulfur:MWCNT =7:3) in ethanol through ball milling (450 rpm, 30 min, 10 cycles). Ethanol was removed after the vacuum infiltration process. The obtained 
MWCNT@S mixture was subsequently heated at $70^{\circ} \mathrm{C}$ for $24 \mathrm{~h}$ to remove any trapped ethanol, followed by heating at $155^{\circ} \mathrm{C}$ for $6 \mathrm{~h}$ to achieve melt diffusion. The asprepared MWCNT@S was combined with the CNF and CNT composite (CNT@CNF) (diameter $=45 \mu \mathrm{m}, \mathrm{KC}$ Flock, Nippon Paper Group, Japan) and SWCNT powder (diameter $=80 \mathrm{~nm}$, Nepes Co., South Korea) through ball milling for $10 \mathrm{~min}$ followed by vacuum infiltration and drying at $60^{\circ} \mathrm{C}$ for $24 \mathrm{~h}$ in a vacuum oven. The CNF-based flexible sulfur cathode was obtained with a controlled thickness $(250-600 \mu \mathrm{m}$, depending on the loading value). The CNT-coated separator was fabricated by a simple solution-casting method. First, a CNT-dispersed solution (SWCNTs, 0.4 wt\% in NMP, Nepes Corp., Korea) was blended with a polyvinylidene fluoride (PVDF) binder (Solvay Corp., Belgium) at a composition of CNT:PVDF = 9:1. The CNT solution was cast onto a dried commercial glass fiber membrane GA55 (Advantec, Ehime, Japan) to a thickness exceeding $30 \mu \mathrm{m}$ and then vacuum dried at $60{ }^{\circ} \mathrm{C}$ for $24 \mathrm{~h}$.

\section{Fabrication of the conventional sulfur cathodes}

A conventional sulfur cathode $(\mathrm{S} / \mathrm{C}$ composite $=7 / 3$ $(\mathrm{w} / \mathrm{w}))$ /polyvinylidene fluoride (PVDF) binder/super-P carbon black (SPB, Alfa Aesar, USA) carbon additive = $70 / 10 / 20(\mathrm{w} / \mathrm{w} / \mathrm{w})$ on an $\mathrm{Al}$ foil current collector, areal sulfur loading $=1.50 \mathrm{mg} \mathrm{cm}^{-2}$ was prepared using a typical slurry casting method. A commercial polyethylene (PE) separator (thickness $=16 \mu \mathrm{m}$, Celgard, USA) and Limetal anode (thickness $=100 \mu \mathrm{m}$ ) were used for the fabrication of conventional Li-S cells.

\section{Fabrication of the Li-S pouch cells}

The cathode, anode, and separator of the pouch-type cells were $30 \times 50,32 \times 52$, and $35 \times 55 \mathrm{~mm} \times \mathrm{mm}$ in sizes, respectively, and Li metal (thickness: $100 \mu \mathrm{m}$ ) was used as the anode. Aluminum foil was attached to the side of the cathode by carbon paste, and a copper foil was attached to the side of the anode by a roll-press machine. These materials provided the lead tabs. A liquid electrolyte (LE) of $1 \mathrm{M}$ lithium bis(trifluoromethylsulfonyl)imide (LiTFSI) in DOL/DME $=1 / 1(\mathrm{v} / \mathrm{v})$ with $1 \mathrm{wt} \% \mathrm{LiNO}_{3}$ additive was used. The ratio of the electrolyte volume to sulfur weight is denoted as the $\mathrm{E} / \mathrm{S}$ ratio in units of $\mathrm{mL} \mathrm{g}^{-1}$. The $\mathrm{E} / \mathrm{S}$ ratio for all cells examined herein was fixed at $15 \mathrm{~mL} \mathrm{~g}^{-1}$. The energy densities of the Li-S cells were obtained by using the formula $m A h g_{\text {cell }}^{-1} \times 2.1 \mathrm{~V}$, where $\mathrm{g}_{\text {cell }}$ is the total mass of the cell, as listed in Table S1 and Fig. S1 in the Supporting Information.

\section{Characterization}

CR2032-type coin and pouch-type Li-S cells were used to analyze the electrochemical characteristics. We employed a liquid electrolyte of $1 \mathrm{M}$ lithium bis(trifluoromethyl sulfonyl)imide (LiTFSI) in 1,3-dioxolane [DOL]/1,2-dimethoxyethane $[\mathrm{DME}]=1 / 1[\mathrm{v} / \mathrm{v}]$ with $1 \mathrm{wt} . \%$ lithium nitrate $\left(\mathrm{LiNO}_{3}\right)$ additive. The cell performance was investigated using a cycle tester (Won-A Tech) at $25^{\circ} \mathrm{C}$ under various charge/discharge conditions in the potential range of $1.5-3.3 \mathrm{~V}$. The structure and physicochemical characteristics of the multi-walled carbon nanotubes and sulfur composite (MWCNT@S) were obtained by X-ray diffraction (XRD, X'Pert PRO MPD, Panalytical) and thermogravimetric analysis (TGA, SDT Q600, TA Instruments). The surface and cross-sectional morphologies of the electrodes were characterized by field-emission scanning electron microscopy (FE-SEM, S-4800, Hitachi) in conjunction with energy-dispersive X-ray spectroscopy (EDS). Postmortem analysis of Li-S cells after the charge/discharge cycling tests was performed using X-ray diffraction (XRD, X'Pert PRO MPD, Panalytical) and X-ray photoelectron spectroscopy (XPS, Escalab250, VGScientific) with nonmonochromatic $\mathrm{Al} \mathrm{K \alpha} \mathrm{X}$-ray radiation $(\mathrm{h} v=14868)$ as the excitation source and a pass (resolution) energy of $50.0 \mathrm{eV}$.

\section{Results and discussion}

\section{Investigation of the fibrous sulfur cathodes}

Because sulfur has an excellent theoretical specific capacity $\left(1675 \mathrm{~mA} \mathrm{~h} \mathrm{~g}^{-1}\right)$ and energy density $(2500 \mathrm{Wh}$ $\left.\mathrm{kg}^{-1}\right)$, it is the primary active material in Li-S batteries. However, pristine sulfur is an insulator in regard to electrical and ionic conductivity, which causes electrodes with pristine sulfur to have low conductivity and a large charge transfer resistance. Therefore, conductive nanomaterials have to be incorporated in sulfur cathodes to obtain high electrical conductivity to achieve Li-S batteries with high electrochemical performance. In this study, we attempted to create a fibrous nonwoven network of sulfur cathodes by combining melt-diffused sulfur in conductive CNTs with CNF supports. Figure 1 shows the stepwise process for fabricating flexible Li-S batteries based on the CNT@CNF-based sulfur cathodes and CNT-coated GA55 glass fiber separators. Briefly, an aqueous suspension of CNFs and single-walled CNTs (SWCNTs) was mechanically mixed to prepare CNT@CNF as a flexible binder. Separately, MWCNT@S was prepared by mixing sulfur powder with MWCNTs by high-energy ball milling and heating at $155^{\circ} \mathrm{C}$ for $6 \mathrm{~h}$ to achieve melt diffusion ${ }^{19,30}$. During the melting process, a sulfur layer with a nanoscale thickness conformably formed on the surface of the one-dimensional structure of the MWCNTs. The formation of MWCNT@S was verified by XRD. In the powder-XRD measurement in Fig. 2a, pure sulfur shows an $\alpha$-sulfur crystal with an orthorhombic structure from the cyclooctasulfur $\left(\mathrm{S}_{8}\right)$ molecules $^{31}$. These XRD peaks were well integrated with those patterns by MWCNTs. This result reveals that elemental sulfur was successfully impregnated in the CNT networks 


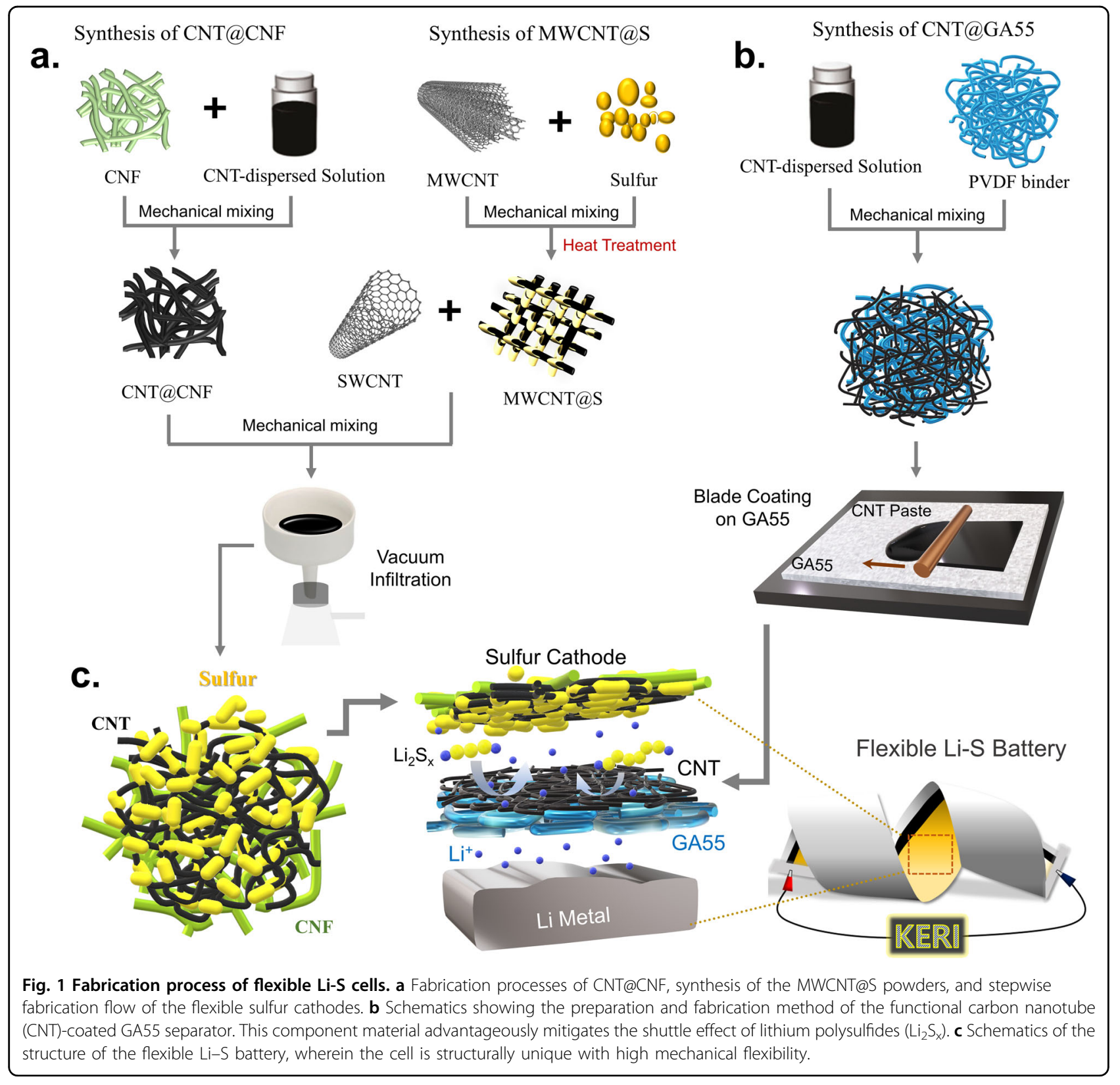

after the melt infiltration process without the agglomeration of sulfur particles. To investigate the sulfur content in the MWCNT@S composites, TGA was carried out under oxidative conditions. Figure $2 \mathrm{~b}$ shows that a significant mass loss was observed in the range of $190-310^{\circ} \mathrm{C}$, which is attributed to the desorption of sulfur and its chemical reaction to $\mathrm{SO}_{2}{ }^{27}$. Therefore, we obtained a relatively high total sulfur content of approximately 65 wt.\% in the MWCNT@S powder.

CNF-based sulfur cathodes were finally fabricated by mixing the as-prepared MWCNT@S with CNT@CNF and SWCNT powder through ball milling and ultrasonication, followed by vacuum infiltration and drying at $60^{\circ} \mathrm{C}$ for $24 \mathrm{~h}$ in a vacuum oven ${ }^{28,32,33}$. The thickness of the sulfur cathodes could be controlled to $250-600 \mu \mathrm{m}$ by changing the loading contents during the vacuum filtration process. The scanning electron microscopy (SEM) and energy-dispersive X-ray spectroscopy (SEM-EDS) images (Fig. 2e, f) show that the CNT@CNF, SWCNTs, and MWCNT@S materials of the composite electrodes were well mixed and uniformly distributed. The weightbased composition ratios in the CNF-based sulfur cathodes were 51.80, 27.0, 8.4, and $12.8 \mathrm{wt} . \%$ for elemental sulfur, MWCNTs, CNT@CNF, and SWCNTs, respectively. Notably, our all-fibrous sulfur cathode did not require a current collector owing to the high electrical 

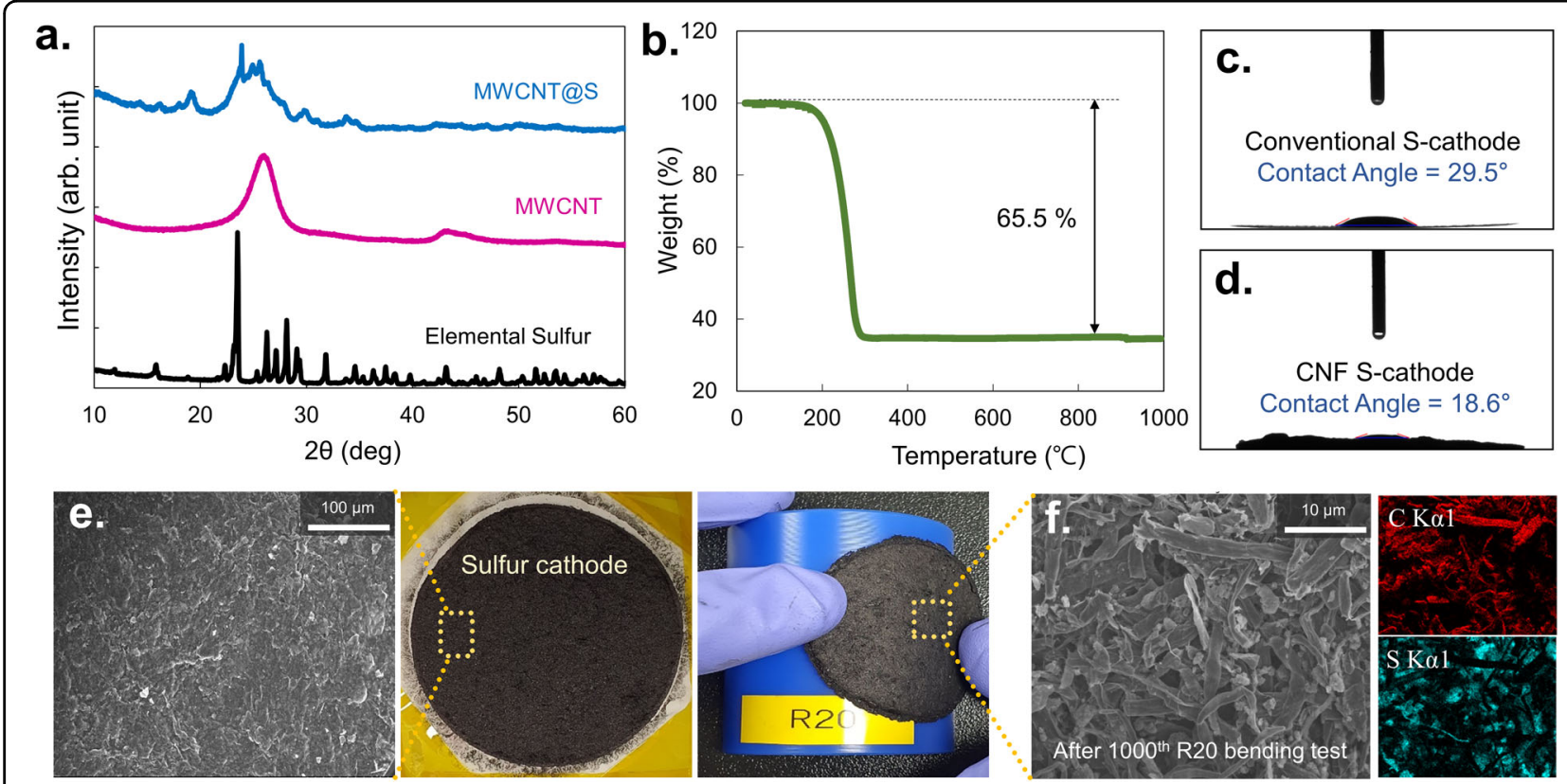

Fig. 2 Characterization of fibrous sulfur cathodes. (a) XRD pattern of MWCNT@S and (b) TGA profiles for quantifying the sulfur content on MWCNT@S, forming the active material. Contact angle measurement of (c) the conventional sulfur cathodes and (d) CNF-based sulfur cathodes. e Scanning electron microscopy (SEM) and digital camera images of the cellulose nanofiber (CNF)-based sulfur cathode (scale bar $=100 \mu \mathrm{m})$. The CNFs are well coated with CNTs, and MWCNT@S is well distributed on the cathode.f SEM and EDS images of the prepared cathodes after 1000 cycles of the R20 bending test.

conductivity of the SWCNTs. The CNT@CNF-based sulfur cathodes showed an approximately 100 times lower sheet resistance $\left(106 \mathrm{~S} \mathrm{sq}^{-1}\right)$ than that of a conventional sulfur cathode $\left(0.7 \mathrm{~S} \mathrm{sq}^{-1}\right)$. The conductive SWCNT network in the sulfur cathodes acted as rapid pathways for electron transfer and conduction through extended $\pi$ conjugated frameworks, consequently enabling the fabrication of current collector-free devices. This mechanism is critical to achieve Li-S batteries with a high energy density because a large amount of sulfur is available within the same electrode thickness in contrast to conventional Li-S battery cells with collectors. Moreover, this thin structural design can offer excellent mechanical flexibility and reliable electrochemical performance when repeatedly bent or folded. To approve the mechanical flexibility of the sulfur cathode, an R20 bending test was implemented for thousands of cycles. As seen in the digital and FE-SEM images in Fig. 2e, f, the fibrous composite electrodes show no noticeable cracks or defects even after the 1000th bending cycle. In addition, the characteristic features of the nonwoven CNF-based sulfur cathode materials with well-developed pores and different diameters induced capillary action to promote the infiltration of liquid electrolytes (LEs) into the electrode. To verify the wettability of the CNF-based sulfur cathode, contact angle measurements were carried out by dropping the LEs on the electrode surface (Fig. 2). The fibrous
CNT@CNF-based sulfur cathode showed a lower contact angle than a conventional cathode used with spherical materials, such as SPB. This result allows the electrolyte to efficiently penetrate mesoporous cathodes that have high loading values or thick films, forming an ion pathway that contributes to the redox kinetics ${ }^{4,34}$.

\section{CNT-coated GA55 separators}

For the fabrication of the CNT-coated GA55 (CNT@GA55) separator, a CNT-dispersed solution in nmethyl-2-pyrrolidone (NMP) was blended with PVDF binder at a composition of CNT:PVDF $=9: 1$ by weight. Subsequently, CNT and PVDF blend solutions were uniformly coated on a dried glass fiber-based commercial separator (GA55) with a controllable thickness of $20-40 \mu \mathrm{m}$ using a cast machine, followed by vacuum drying at $60^{\circ} \mathrm{C}$ for $24 \mathrm{~h}$. Figure 3a shows the crosssectional SEM images of the assembly of a separator with the SWCNT nonwoven network, in which the conductive CNT layer has a thickness of $\sim 30 \mu \mathrm{m}$. The CNT@GA55 separator had two different faces: a black (front) side with CNTs and a white (back) side, which was the pristine separator without CNTs. As shown in Fig. 3b and $3 \mathrm{c}$, many small pores with pore sizes ranging from 550 to $750 \mathrm{~nm}$ are found on the SWCNT-coated face, which are much smaller than those on the as-received GA55 $(5.5-10 \mu \mathrm{m})$. While these porous network 

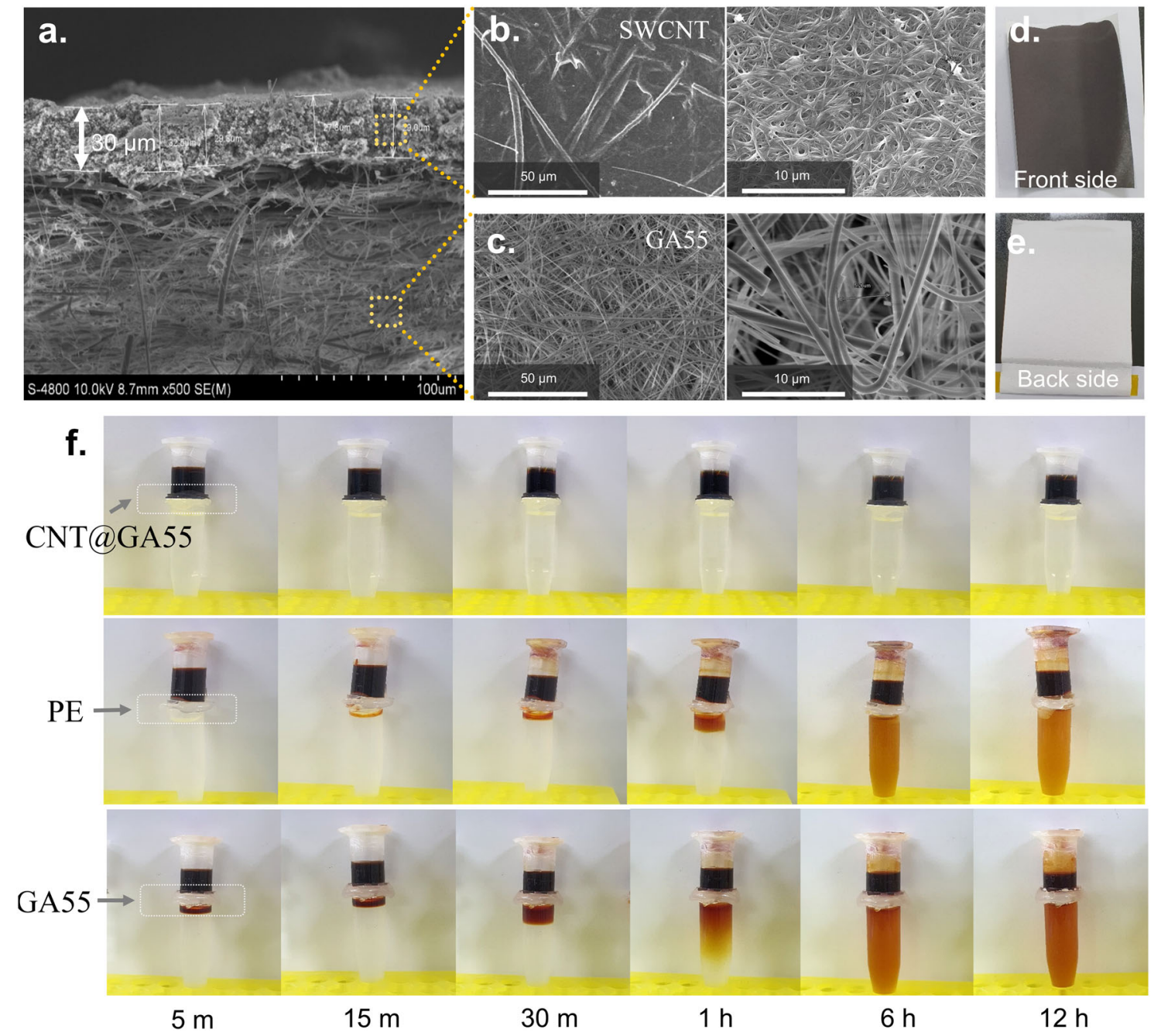

Fig. 3 Structures and permeation behavior of CNT-coated glass fiber membrane. a SEM images of a cross-section (scale bars $=100 \mu \mathrm{m})$ and the surfaces of the (b) CNT-coated GA55 and (c) pristine GA55 separation membranes. The pores are denser on the CNT-coated surface than on pristine GA55 (scale bars $=50 \mu \mathrm{m}$ and $10 \mu \mathrm{m}$ in surface images). $\mathbf{d}$, e Photographs of the CNT-coated front side and back side of the pristine separator. f Photographs show the permeation behavior of polysulfides through various separators, such as the CNT@GA55, commercial polyethylene $(\mathrm{PE})$, and pristine GA55 separators.

structures play a role in conducting ionic species after being filled with LEs, small submicron pores in the CNTcoated layer are expected to suppress the migration of $\mathrm{Li}_{2} \mathrm{~S}_{\mathrm{x}}$ to the anode $\mathrm{e}^{35-38}$. To verify the effect of the CNTcoated separator on preventing the "shuttle effect", the permeation behavior of $\mathrm{Li}_{2} \mathrm{~S}_{\mathrm{x}}$ in LEs through various separators (i.e., CNT@GA55, commercial polyethylene (PE) with a thickness of $20 \mu \mathrm{m}$, and the as-received GA55 without a CNT coating) was monitored as a function of time. Figure $3 \mathrm{f}$ shows a simple experimental setup for easy visualization. The separators were placed between a polysulfide solution (upper side, $0.1 \mathrm{M} \mathrm{Li}_{2} \mathrm{~S}_{8}$ solution in $\mathrm{DOL} / \mathrm{DME}=1 / 1[\mathrm{v} / \mathrm{v}]$ ) and a solvent mixture (bottom side, $\mathrm{DOL} / \mathrm{DME}=1 / 1[\mathrm{v} / \mathrm{v}])$. No appreciable color change was observed for the CNT-coated GA55 separator even after $12 \mathrm{~h}$, whereas a solution were colored reddish-brown with respect to time for the pristine GA55 and conventional PE separators. This result demonstrated that the CNT@GA55 separator effectively suppressed $\mathrm{Li}_{2} \mathrm{~S}_{\mathrm{x}}$ migration. The polysulfides that were dissolved in the electrolytes were effectively trapped by the submicron pores of the CNT networks as well as the Coulombic interaction with a negative surface charge on the SWCNTs ${ }^{4,28,29,35,36}$. Notably, as compared with a PE separator, the pristine GA55 showed more permeation of polysulfides, presumably due to a relatively large pore size of GA55. However, the permeation behavior inside the Li$S$ battery cell was clearly different from those under extreme situations. Moreover, when the PE separator was used, peeling or defects occurred due to the low physical 

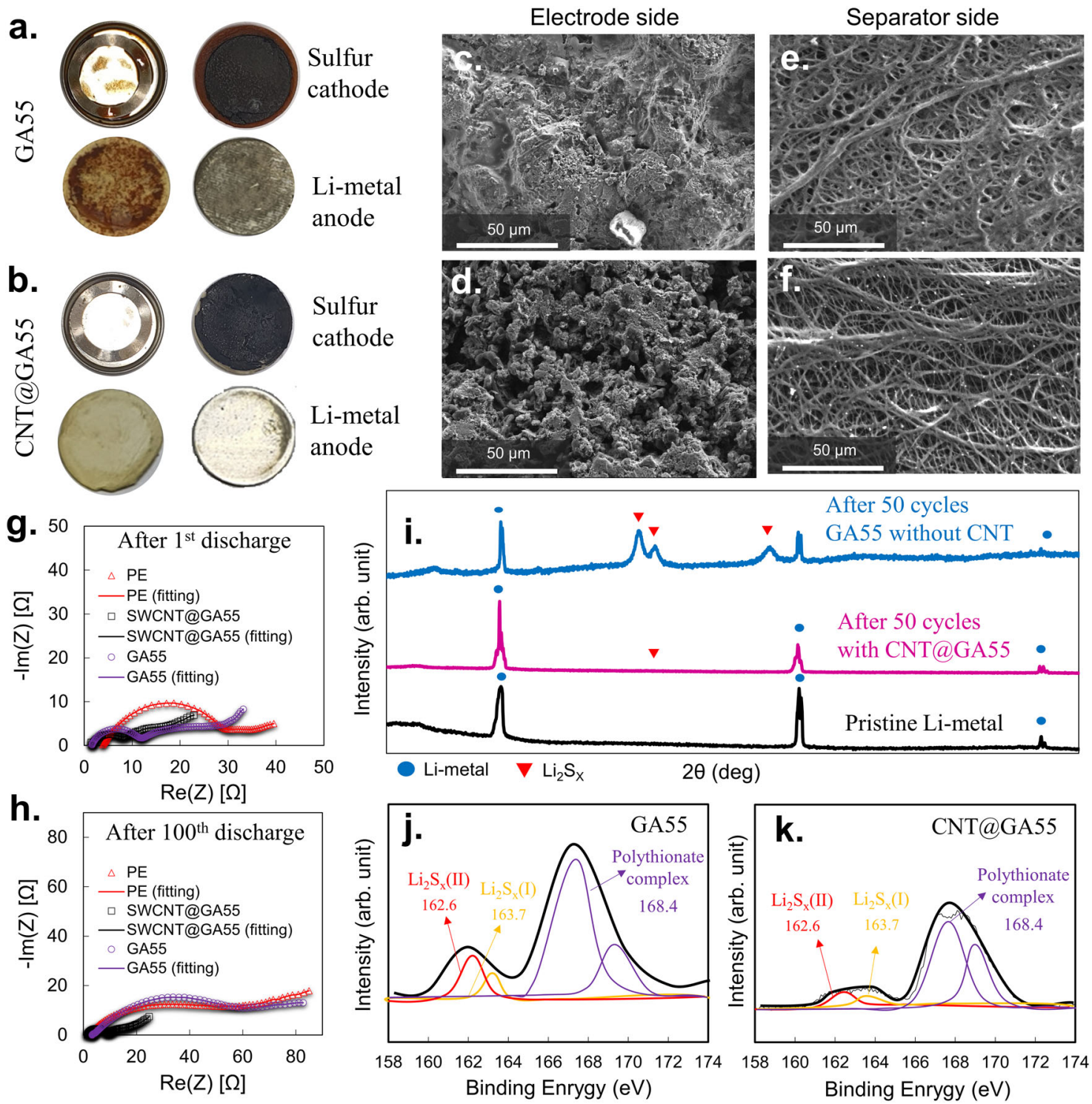

Fig. 4 Cycling stability of Li-S cells. a, b Photographs of the disassembled Li-S cells after 20 charge/discharge cycles. a The pristine GA55 separator without the CNT coating changes to a reddish-brown color after the infusion of lithium-polysulfide through the GA55 membrane; in contrast, (b) the cell with the CNT interlayer exhibits no appreciable color change. c-f FE-SEM images of the anode and CNT@GA55 separator sides after 50 charge/ discharge cycles: (c) Li-metal without the CNT interlayer and (d) Li-metal with the CNT interlayer and (e) separator without the CNT interlayer and (f) separator with the CNT interlayer. (g) EIS Nyquist plots after the 1st discharge and (h) after the 100th discharge. i XRD patterns of pristine Li-metal and GA55 separators with and without the CNT interlayer after 50 charge/discharge cycles. The $\mathrm{Li}_{2} \mathrm{~S}_{x}$ peaks are labeled. S $2 \mathrm{p}$ XPS spectrum (j) of the pristine GA55 separator (facing the Li-metal anode) after 50 cycles and (k) of the GA55 separator with an SWCNT coating (facing the Li-metal anode) after 50 cycles.

bonding strength between the coating materials and PE. On the other hand, GA55 maintained strong physical bonding with the coating materials, thereby enabling the high mechanical flexibility of our Li-S cells.

For applying to flexible Li-S batteries, the CNT interlayer coated on the separator must exhibit robust performance for inhibiting the migration of dissolved $\mathrm{Li}_{2} \mathrm{~S}_{\mathrm{x}}$ in the electrolyte even under mechanical stress conditions. In this regard, the surfaces of the separator and anode sides were analyzed after disassembling the fabricated Li-S cells after 20 charge/discharge cycles. Figure 4a, b shows photographs of the disassembled Li-S cells after 20 cycles based on the pristine GA55 and CNT-coated GA55 separators, respectively. The GA55 separator without the CNT coating showed a significant color change from white to reddish-brown due to the infusion of $\mathrm{Li}_{2} \mathrm{~S}_{\mathrm{x}}$ through the GA55 membrane, while that of the Li-S cell containing the CNT@GA55 separator exhibited 
clean $\mathrm{Li}$ metal and a separator with no $\mathrm{Li}_{2} \mathrm{~S}_{\mathrm{x}}$ contamination. From the FE-SEM images of the $\mathrm{Li}$ anode and separator side facing the Li-metal anode after 50 charge/ discharge cycles (Fig. 4c-f), it was also confirmed that the surfaces of both the electrode and separator sides without the CNT interlayer were significantly roughened by the surface deposition of low-valence state lithium sulfides, such as $\mathrm{Li}_{2} \mathrm{~S}_{2}$ or $\mathrm{Li}_{2} \mathrm{~S}$; in contrast, this phenomenon was not observed with the CNT-coated GA55 cells. The intermediate redox products $\mathrm{Li}_{2} \mathrm{~S}_{\mathrm{x}}$ in a high-valence state were readily soluble in the LEs, and these polysulfide ions migrated toward the $\mathrm{Li}$ anode. After the reaction of polysulfide ions with $\mathrm{Li}$ metal and the deposition of solidphase $\mathrm{Li}_{2} \mathrm{~S}_{2}$ or $\mathrm{Li}_{2} \mathrm{~S}$, the active sulfur materials were reduced, causing a decrease in the Coulombic efficiencies of the battery cells. Therefore, it was revealed that the CNT interlayer was very effective in suppressing the migration of polysulfides toward Li-metal anodes to prevent shuttle phenomena.

A series of electrochemical measurements were carried out for half cells after the first discharge and after 100 discharge cycles. For comparison, CNT@CNF-based sulfur cathodes with SWCNT-coated GA55 membranes, commercial PE, and pristine GA55 separators were tested with electrochemical impedance spectroscopy (EIS) measurements to characterize the internal resistance and charge transfer process ${ }^{5}$. Figure S2 and Table S2 in the Supporting Information show the equivalent circuit model and corresponding results to determine the resistance components. It should be noted that after the first discharge, the cells based on SWCNT-coated separators showed much smaller charge transfer resistance $\left(R_{c t}\right)$ values than those based on the pristine GA55 and PE separators (Fig. $4 \mathrm{~g}$ ). This result indicated the improved charge transfer properties of the electrodes, attributable to the conductive CNT networks that provided rapid pathways for electron transport. Moreover, the EIS spectra also showed that the SWCNT-coated GA55 separator maintained a low $\mathrm{R}_{\mathrm{ct}}$ after 100 cycles. In comparison, the PE and GA55 separators exhibited a significant increase in $R_{c t}$ after 100 discharge cycles due to the deposition of insulating solid phases after reactions with $\mathrm{Li}$ metal (Fig. 4h). These results confirmed that the SWCNT interlayer efficiently suppressed the migration of lithium polysulfides $^{39}$. The surface of the separator facing the Li-metal anode was analyzed by XRD. Figure 4i shows the XRD patterns of the pristine Li-metal anode and those with GA55 without CNT and CNT@GA55 separators after 20 discharge cycles. While the $\mathrm{Li}_{2} \mathrm{~S}_{\mathrm{x}}$ peaks at $2 \theta=43^{\circ}, 44^{\circ}$, and $51^{\circ}$ were hardly detected at the anode of the cell with the CNT interlayer, those peaks appeared when the CNT interlayer was absent in the separator ${ }^{40-42}$. For the analysis of chemical species on the surface of the GA55 separator, the membrane facing the side with the
Li-metal anode was measured using X-ray photoelectron spectroscopy (XPS), as shown in Fig. 4j, f. In the XPS spectra, the $\mathrm{S} 2 \mathrm{p}_{3 / 2}$ peaks at 162.6 and $163.7 \mathrm{eV}$ corresponded to lower-order polysulfides $\mathrm{Li}_{2} \mathrm{~S}_{\mathrm{x}}$ (II) and higherorder polysulfides $\mathrm{Li}_{2} \mathrm{~S}_{\mathrm{x}}(\mathrm{I})$, respectively ${ }^{24}$. In addition, the $\mathrm{S} 2 \mathrm{p}_{3 / 2}$ peak at $168.4 \mathrm{eV}$ is known to represent the polythionate complex ${ }^{43}$. The CNT@GA55 samples exhibited much smaller lithium polysulfide peaks than the pristine GA55 membrane, suggesting that the CNT interlayer could suppress the migration of polysulfide by trapping it in small submicrometer pores and, partially, by the GibbsDonnan effect due to the surface charges on the CNT networks.

\section{Electrochemical performance of the flexible Li-S cells}

The combination of the CNT@CNF-based sulfur cathode and CNT-coated GA55 separator enabled a cell with significantly improved electrochemical performance compared to that with conventional sulfur cathodes and separators. Figure 5a shows the cycling performance profiles in regard to the discharge capacity and Coulombic efficiency (at a charge/discharge current density of $1.57 \mathrm{mAh} \mathrm{cm}^{-2}$ ) of the flexible Li-S cells with the CNT@CNF-based sulfur cathode and GA55 separator before and after its functionalization with the SWCNT interlayer. The Li-S battery containing the CNT interlayer on the GA55 separator showed excellent 50cycle stability compared to a typical GA55 separator at the same C-rate $(0.1 \mathrm{C})$. The high discharge capacity of more than $800 \mathrm{mAh} \mathrm{g}^{-1}$ mainly resulted from fast redox kinetics stemming from the nanoscale fibrous network for electron transfer, conduction pathways formed by the SWCNTs and MWCNTs, and easy access to $\mathrm{Li}$ ions in the LEs. At C-rates of $0.5 \mathrm{C}$ and $1.0 \mathrm{C}$, our Li-S cells also showed good cycling stabilities. Notably, the Li-S battery based on the CNT@CNF cathode and CNT@GA55 separator with an initial specific capacity of approximately $667 \mathrm{mAh} \mathrm{g}^{-1}$ at $0.5 \mathrm{C}$ exhibited a slow decay rate of $<1 \%$ per cycle after 100 cycles (Fig. 5b). The superior cycling retention characteristics of the CNT@GA55 cells are primarily attributed to the effective suppression of the shuttle phenomena of dissolved $\mathrm{Li}_{2} \mathrm{~S}_{\mathrm{x}}$. It can be verified that the CNT-coated separator mitigated the crossover of polysulfides, subsequently improving the capacity retention during cycling processes. Because CNT-coated GA55 decreased the pore size of the separator from $5 \mu \mathrm{m}$ to $900 \mathrm{~nm}$ and the electrostatic interactions with the CNT networks, the migration of $\mathrm{Li}_{2} \mathrm{~S}_{\mathrm{x}}$ during ion transport ensured cycling stability.

Figure $5 \mathrm{c}$ shows the charge and discharge rate capabilities of Li-S cells assembled with the flexible sulfur cathode and CNT-functionalized GA55 membrane. The cycling performance of the cells was examined at different discharge current densities of 1.57, 7.87, 15.74, and $31.48 \mathrm{mAh} \mathrm{cm}^{-2}$, which demonstrated discharge capacities of $940,656,592$, and $457 \mathrm{mAh} \mathrm{g}^{-1}$, respectively. The 

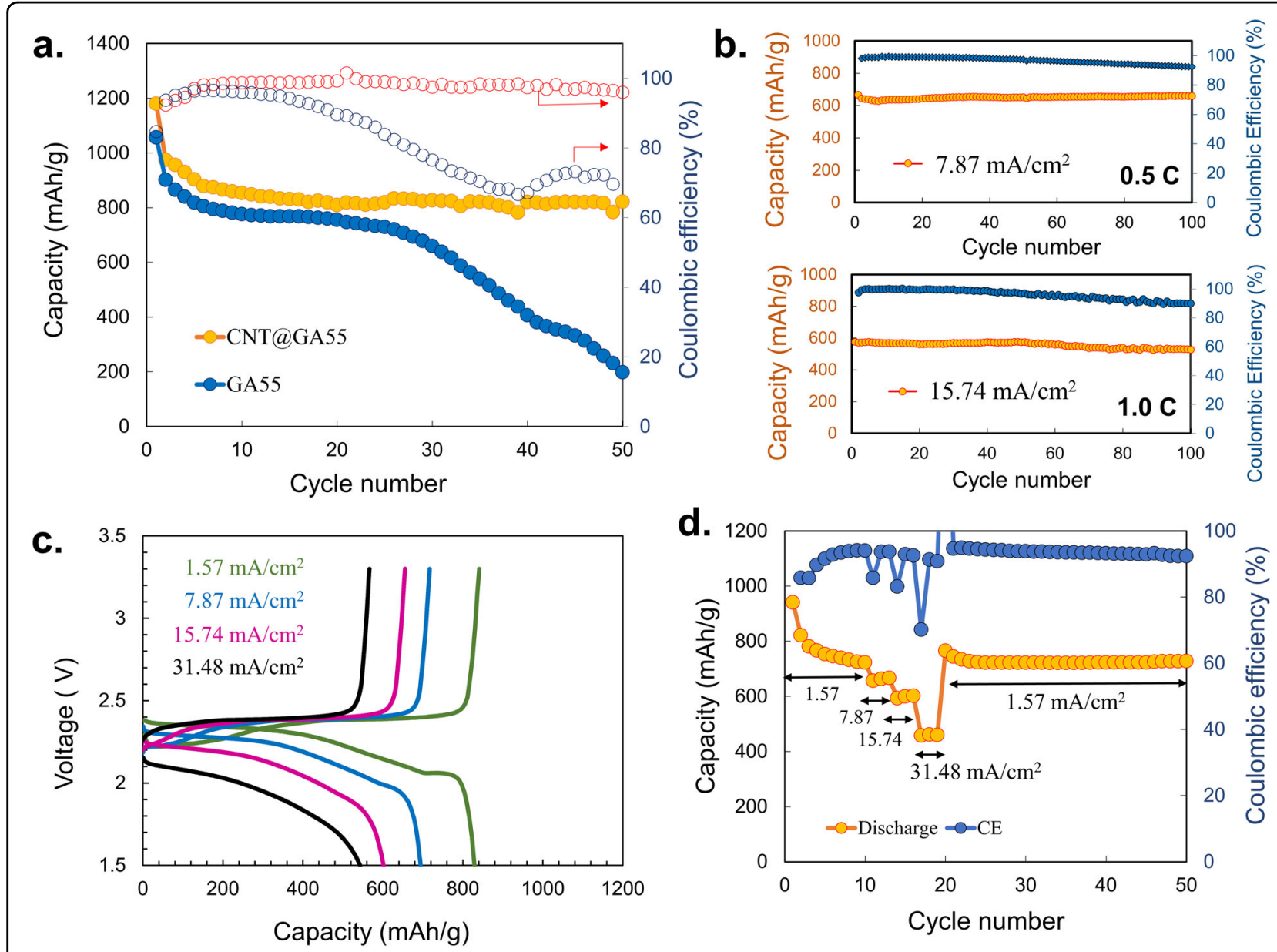

Fig. 5 Electrochemical performance of Li-S cells. a Discharge capacity and Coulombic efficiency of Li-S cells based on a CNF-based sulfur cathode and a commercial GA55 separator with and without a CNT-interlayer coating. The cycling test was performed at a charge/discharge current density of $1.57 \mathrm{mAh} \mathrm{cm}^{-2}$. b Cycling performance profiles of the Li-S cells at various C-rates and at 7.87 and $15.74 \mathrm{mAh} \mathrm{cm}^{-2}$. c Galvanostatic charge/ discharge profiles and (d) discharge rate capabilities of the Li-S cells with a CNF-based sulfur cathode and a CNT-interlayer coated GA55 separator over a wide range of discharge current densities (1.57-31.48 $\left.\mathrm{mAh} \mathrm{cm}^{-2}\right)$ at a fixed charge current density $\left(1.57 \mathrm{mAh} \mathrm{cm}^{-2}\right)$ and a voltage range of $1.5-3.3 \mathrm{~V}$ (sulfur loading $=8-9 \mathrm{mg} \mathrm{cm}^{-2}$ ).

discharge capabilities of the cells were also confirmed at different C-rates (Fig. 5b), where the discharge density was varied from 7.87 to $15.74 \mathrm{mAh} \mathrm{cm}^{-2}$ at a fixed charge current density of $1.57 \mathrm{mAh} \mathrm{cm}^{-2}$. Notably, during 100 cycles, the discharge capacities were maintained stably at 659 and $571 \mathrm{mAh} \mathrm{g}^{-1}$ at current densities of 7.87 and $15.74 \mathrm{mAh} \mathrm{cm}^{-2}$, respectively. As shown in Fig. $5 \mathrm{~d}$, even at a high C-rate, the Li-S cells based on the CNT@CNFbased sulfur cathodes and CNT interlayer-coated GA55 separator-assembled cells worked well owing to their high sulfur-loading value of $8.5 \mathrm{mg} \mathrm{cm}^{-2}$. As the fibrous composite electrodes contained no traditional massive metallic current collector, their total areal weight was considerably lower than that of a sulfur cathode with the same area in a conventional Li-S cell. Consequently, the CNT@CNF-based sulfur cathode showed a remarkably higher sulfur loading per cell weight than a sulfur cathode in a conventional $\mathrm{Li}-\mathrm{S}$ cell.

\section{Mechanical flexibility of Li-S batteries}

For the mechanical flexibility tests, we fabricated $30 \times 50$ pouch-type Li-S cells based on a CNT@CNF-based sulfur cathode and CNT-coated GA55 separator. A test kit using 46 light-emitting diodes was assembled to visually confirm the stable operation of the flexible battery under various deformation conditions (e.g., bending by $90^{\circ}$, folding by $180^{\circ}$, rolling, and crumpling). As shown in Fig. 6a, our flexible Li-S battery successfully powered the LED module, even under severely deformed (crumpled) states. Figure 6b shows the electrochemical performance of the flexible battery under repeated deformation conditions with an R20 bending tester. An initial high discharge capacity of 

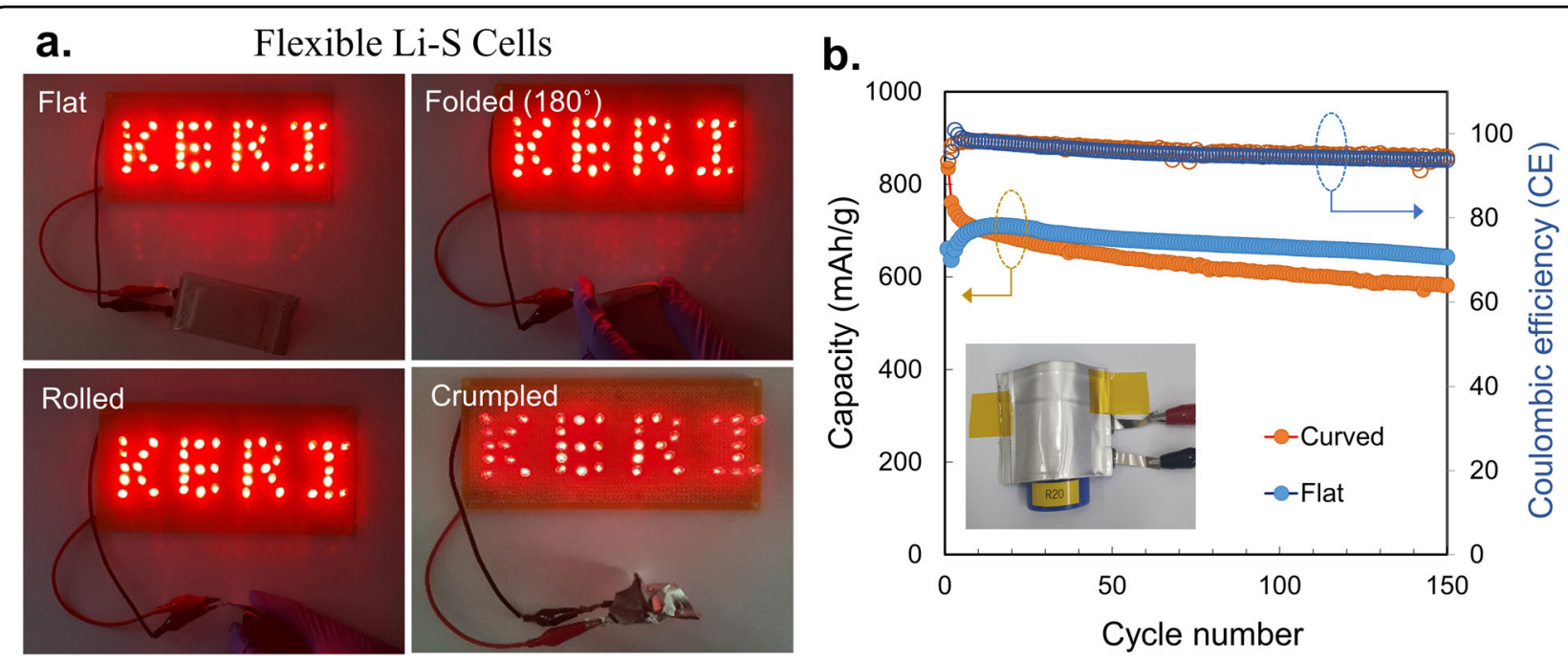

Fig. 6 Characterization fo Flexible Li-S battery. Digital camera images showing a red light-emitting diode (LED) logo lit by (a) a flat and bent Li-S battery (bent states: folded $\left(180^{\circ}\right)$, rolled, and crumpled) and (b) cycling performance of the flexible Li-S battery under flat and bent conditions (current density $=1.06 \mathrm{mAh} \mathrm{cm}^{-2}$ ).

$834 \mathrm{mAh} \mathrm{g}^{-1}$ and a Coulombic efficiency at a current density of $1.06 \mathrm{mAh} \mathrm{cm}^{-2}$ were stably maintained (Fig. $6 \mathrm{~b}$ ). The excellent mechanical flexibility and stable cycling performance of our Li-S cells were primarily attributed to the highly conductive and free-standing all-fiber-based sulfur cathodes that could withstand mechanical stress and enabled the intimate assembly of SWCNT interlayers and glass fiber membranes. The CNT networks in the composite electrodes played a crucial role in providing an efficient pathway for electron transport and high electrical conductivities as well as enabling a high sulfur loading content, thereby achieving a high energy density. Moreover, the CNT-coated GA55 separator prevented leakage current even in a severe mechanically bent state and simultaneously suppressed the shuttle effect of polysulfides.

Our flexible Li-S cells based on the CNT@CNF-based fibrous sulfur cathode and CNT-interlayer-modified GA55 separator had many advantages over a conventional Li-S battery in terms of material selection and structural features: (i) The CNT@CNF-based sulfur cathode had high electrical conductivity due to the formation of a conductive CNT network; therefore, this cathode did not require a current collector and could contain a large amount of sulfur within the same thickness as a conventional Li-S cathode, thereby achieving a high energy density. (ii) The CNT@GA55 separator was prepared by a simple solution process, and the CNT interlayer coated on the commercial membrane played an essential role in mitigating the shuttle effect of $\mathrm{Li}_{2} \mathrm{~S}_{\mathrm{x}}$, thereby ensuring cycling stability. The negatively charged chemical species dissolved in the organic LEs were blocked by the small $(<1 \mu \mathrm{m}) \mathrm{CNT}$ pores and were repelled by electrostatic forces due to the negative charges in the CNT basal plane. (iii) The three-dimensional fibrous structure of both the sulfur cathode and CNT-coated membrane provided twochannel continuous pathways for electrons and $\mathrm{Li}$ ions, contributing to the fast redox kinetics of the reversible charge and discharge electrochemical reactions ${ }^{4}$. (iv) The hydrophilic surface of the CNFs supported conformable CNT network formation and provided superior LE wettability. (v) Finally, the CNF-based fibrous structures maintained their mechanical flexibility and robustness without using a polymeric binder. This result could prevent unnecessary side reactions and decrease the specific capacity caused by insulating polymer binders ${ }^{40,44,45}$. As shown in Fig. S3 and Table S3 in the Supporting Information, it can be confirmed that our flexible Li-S battery based on fibrous $600 \mu \mathrm{m}$ thick sulfur cathodes and CNTcoated GA55 separators had the highest capacity of 8.4 $\mathrm{mAh} \mathrm{cm} \mathrm{cm}^{-2}$ at a high sulfur loading of $\sim 10.4 \mathrm{mg} \mathrm{cm}^{-2}$ and a rate of $0.1 \mathrm{C}$. When the thickness of the sulfur cathodes was reduced to 475 and $350 \mu \mathrm{m}$, the areal capacity was still high at 6.68 and $4.37 \mathrm{mAh} \mathrm{cm}^{-2}$ with sulfur loadings of 8.1 and $5.2 \mathrm{mg} \mathrm{cm}^{-2}$ at $0.1 \mathrm{C}$, respectively. Figure $\mathrm{S} 4$ in the Supporting Information shows a chart comparing the energy densities of the prepared Li-S batteries with other types of battery categories, and the red star shows the electrochemical performance of our cell in this study. This table reveals that our Li-S battery exhibited excellent gravimetric and volumetric energy densities, which enabled us to fabricate lightweight and compact energy storage devices. The high discharge capacity and good cycling endurance of our flexible Li-S batteries show that they could be used as high-energy-density flexible energy storage media, thereby achieving flexible electronic products and wearable smart devices. 


\section{Conclusions}

We presented flexible high-energy-density Li-S batteries for the application of next-generation flexible and wearable electronics. The Li-S cell was assembled with a CNF-based sulfur cathode and a CNT-coated GA55 commercial separator. We designed all-fibrous composite cathodes without an insulating polymeric binder and heavy metallic current collector and selected simple and low-cost materials. The conductive CNT network provided high electrical conductivity and a high sulfur content in the electrode, enabling a high energy density. The SWCNT-coated GA55 membrane effectively suppressed the shuttle effect of polysulfides due to its small pores and $\mathrm{Li}_{2} \mathrm{~S}_{\mathrm{x}}$ repulsion due to functionalized nanocarbon materials. The fabricated Li-S batteries exhibited significantly improved electrochemical performance in terms of redox kinetics and cycling stability compared to that of conventional Li-S cells. Our Li-S cells showed stable capacities of $854 \mathrm{mAh} \mathrm{g}^{-1}$ over 50 cycles and achieved a high gravimetric energy density of $443.4 \mathrm{Wh}$ $\mathrm{kg}^{-1}$ per cell. In addition to their high energy density, they demonstrated mechanical flexibility even under severe deformation (crumpled) conditions and stability with no observed short circuits between the two electrodes. Moreover, the material selection and design strategies used in our study are cost effective and utilize a simple fabrication process, making the resulting materials good candidates for commercialization. The proposed CNF- and CNT-based composite electrodes and nanocarbon-modified separator of Li-S batteries may be a promising solution for developing advanced next-generation flexible batteries.

\section{Acknowledgements \\ This research was supported in part by the Korea Electrotechnology Research Institute (KERI) (No. 21A01016) and by grants from the National Research Foundation of Korea (NRF), which are funded by the Korean government (No. 2020R1F1A1071718 and No. 2020R1A4A1019463) and the open lab program for regional specialized technology development and diffusion funded by the Busan Innovation Institute of Industry, Science \& Technology Planning (BISTEP) in 2020. The SWCNT powder and CNT solution were obtained from Nepes Corp., South Korea.}

\section{Author details \\ ${ }^{1}$ Next-Generation Battery Research Center, Korea Electrotechnology Research Institute, 12 Bulmosan-ro 10beon-gil, Seongsan-gu, Changwon-si, Gyeongsangnam-do 51543, Republic of Korea. ${ }^{2}$ Department of Smart and Green Technology Engineering, Pukyong National University, 45 Yongso-ro, Nam-gu, Busan 48513, Republic of Korea. ${ }^{3}$ Analysis and Assessment Center, Research Institute of Industrial and Science Technology, 67 Cheongam-ro, Nam-gu, Pohang-si, Gyeongsangbuk-do 37673, Republic of Korea. ${ }^{4}$ Department of Materials Science and Engineering, Gachon University, 1342 Seongnam-daero, Sujeong-gu, Seongnam-si, Gyeonggi-do 13120, Republic of Korea. ${ }^{5}$ Department of Nanotechnology Engineering, Pukyong National University, 45 Yongso-ro, Nam-gu, Busan 48513, Republic of Korea}

\section{Conflict of interest}

The authors declare no competing interests.

\section{Publisher's note}

Springer Nature remains neutral with regard to jurisdictional claims in published maps and institutional affiliations.
Supplementary information The online version contains supplementary material available at https://doi.org/10.1038/s41427-021-00295-y.

Received: 8 December 2020 Revised: 4 February 2021 Accepted: 8 February 2021.

Published online: 2 April 2021

\section{References}

1. Peake, J. M., Kerr, G. \& Sullivan, J. P. A critical review of consumer wearables, mobile applications, and equipment for providing biofeedback, monitoring stress, and sleep in physically active populations. Front. Physiol. 9, 1-19 (2018).

2. Aroganam, G., Manivannan, N. \& Harrison, D. Review on wearable technology sensors used in consumer sport applications. Sens. (Basel) 19, 1983 (2019).

3. Song, W.-J. et al. Recent progress in stretchable batteries for wearable electronics. Batteries Supercaps 2, 181-199 (2019).

4. Kim, J.-H. et al. Nanomat Li-S batteries based on all-fibrous cathode/separator assemblies and reinforced Li metal anodes: towards ultrahigh energy density and flexibility. Energy Environ. Sci. 12, 177-186 (2019).

5. Zhou, G. et al. A flexible sulfur-graphene-polypropylene separator integrated electrode for advanced Li-S batteries. Adv. Mater. 27, 641-647 (2015).

6. Ji, X., Lee, K. T. \& Nazar, L. F. A highly ordered nanostructured carbon-sulphur cathode for lithium-sulphur batteries. Nat. Mater. 8, 500-506 (2009).

7. Park, J.-W., Ueno, K., Tachikawa, N., Dokko, K. \& Watanabe, M. ionic liquid electrolytes for lithium-sulfur batteries. J. Phys. Chem. C. 117, 20531-20541 (2013).

8. Baeg, K.-J., Caironi, M. \& Noh, Y.-Y. Toward printed integrated circuits based on unipolar or ambipolar polymer semiconductors. Adv. Mater. 25, 4210-4244 (2013).

9. Koo, J. H., Kim, D. C., Shim, H. J., Kim, T.-H. \& Kim, D.-H. Flexible and stretchable smart display: materials, fabrication, device design, and system integration. Adv. Funct. Mater. 28, 1801834 (2018).

10. Zeng, L., Qiu, L. \& Cheng, H.-M. Towards the practical use of flexible lithium ion batteries. Energy Storage Mater. 23, 434-438 (2019).

11. Manthiram, A., Fu, Y., Chung, S.-H., Zu, C. \& Su, Y.-S. Rechargeable lithium-sulfur batteries. Chem. Rev. 114, 11751-11787 (2014).

12. Li, W., Lee, S. \& Manthiram, A. High-nickel NMA: a cobalt-free alternative to NMC and NCA cathodes for lithium-ion batteries. Adv. Mater. 32, 2002718 (2020).

13. Park, J.W. et al. Solvent effect of room temperature ionic liquids on electrochemical reactions in lithium-sulfur batteries. J. Phys. Chem. C. 117, 4431-4440 (2013).

14. Ueno, $K$. et al. Anionic effects on solvate ionic liquid electrolytes in rechargeable lithium-sulfur batteries. J. Phys. Chem. C. 117, 20509-20516 (2013).

15. Zhang, C. et al. Chelate effects in glyme/lithium bis(trifluoromethanesulfonyl) amide solvate ionic liquids, part 2: importance of solvate-structure stability for electrolytes of lithium batteries. J. Phys. Chem. C. 118, 17362-17373 (2014).

16. Tachikawa, N. et al. Reversibility of electrochemical reactions of sulfur supported on inverse opal carbon in glyme-Li salt molten complex electrolytes. Chem. Commun. 47, 8157-8159 (2011).

17. Kang, W. et al. A review of recent developments in rechargeable lithium-sulfur batteries. Nanoscale 8, 16541-16588 (2016).

18. Dokko, K. et al. Solvate ionic liquid electrolyte for Li-S batteries. J. Electrochem. Soc. 160, A1304-A1310 (2013).

19. Li, L., Wu, Z., Yuan, S. \& Zhang, X.-B. Advances and challenges for flexible energy storage and conversion devices and systems. Energy Environ. Sci. 7, 2101-2122 (2014).

20. Choi, K-H. et al. Heterolayered, one-dimensional nanobuilding block mat batteries. Nano Lett. 14, 5677-5686 (2014)

21. Leijonmarck, S., Cornell, A., Lindbergh, G. \& Wågberg, L. Single-paper flexible Liion battery cells through a paper-making process based on nano-fibrillated cellulose. J. Mater. Chem. A 1, 4671-4677 (2013).

22. Hagen, M. et al. Lithium-sulfur cells: the gap between the state-of-the-art and the requirements for high energy battery cells. Adv. Energy Mater. 5, 1401986 (2015).

23. Wild, M. et al. Lithium sulfur batteries, a mechanistic review. Energy Environ. Sci. 8, 3477-3494 (2015)

24. Zu, C. \& Manthiram, A. High-performance Li/dissolved polysulfide batteries with an advanced cathode structure and high sulfur content. Adv. Energy Mater. 4, 1400897 (2014). 
25. Elazari, R., Salitra, G., Garsuch, A., Panchenko, A. \& Aurbach, D. Sulfurimpregnated activated carbon fiber cloth as a binder-free cathode for rechargeable Li-S batteries. Adv. Mater. 23, 5641-5644 (2011).

26. Hagen, M. et al. Development and costs calculation of lithium-sulfur cells with high sulfur load and binder free electrodes. J. Power Sour. 224, 260-268 (2013).

27. Thieme, S. et al. High capacity micro-mesoporous carbon-sulfur nanocomposite cathodes with enhanced cycling stability prepared by a solventfree procedure. J. Mater. Chem. A 1, 9225-9234 (2013).

28. Kim, J.-H. et al. Functionalized nanocellulose-integrated heterolayered nanomats toward smart battery separators. Nano Lett. 16, 5533-5541 (2016).

29. Xiao, P., Bu, F., Yang, G., Zhang, Y. \& Xu, Y. Integration of graphene, nano sulfur, and conducting polymer into compact, flexible lithium-sulfur battery cathodes with ultrahigh volumetric capacity and superior cycling stability for foldable devices. Adv. Mater. 29, 1703324 (2017)

30. Wang, $X$. et al. Flexible energy-storage devices: design consideration and recent progress. Adv. Mater. 26, 4763-4782 (2014).

31. Liu, G., Niu, P., Yin, L. \& Cheng, H.-M. a-sulfur crystals as a visible-light-active photocatalyst. J. Am. Chem. Soc. 134, 9070-9073 (2012).

32. Yuan, Z. et al. Hierarchical free-standing carbon-nanotube paper electrodes with ultrahigh sulfur-loading for lithium-sulfur batteries. Adv. Funct. Mater. 24 6105-6112 (2014)

33. Kang, H.-S. \& Sun, Y.-K. Freestanding bilayer carbon-sulfur cathode with function of entrapping polysulfide for high performance Li-S batteries. Adv. Funct. Mater. 26, 1225-1232 (2016).

34. Kim, M.-J. et al. Facile fabrication of solution-processed solid-electrolytes for high-energy-density all-solid-state-batteries by enhanced interfacial contact. Sci. Rep. 10, 11923 (2020).
35. Yu, M., Wang, Z., Wang, Y., Dong, Y. \& Qiu, J. Freestanding flexible Li2S paper electrode with high mass and capacity loading for high-energy Li-S batteries. Adv. Energy Mater. 7, 1700018 (2017).

36. Albertus, P., Babinec, S. Litzelman, S. \& Newman, A. Status and challenges in enabling the lithium metal electrode for high-energy and low-cost rechargeable batteries. Nat. Energy 3, 16-21 (2018).

37. Mao, Y. et al. Foldable interpenetrated metal-organic frameworks/carbon nanotubes thin film for lithium-sulfur batteries. Nat. Commun. 8, 14628 (2017).

38. Ahn, W. et al. Interaction mechanism between a functionalized protective layer and dissolved polysulfide for extended cycle life of lithium sulfur batteries. J. Mater. Chem. A 3, 9461-9467 (2015).

39. Wang, $Z$. et al. Constructing metal-free and cost-effective multifunctional separator for high-performance lithium-sulfur batteries. Nano Energy $\mathbf{5 9}$, 390-398 (2019).

40. Scheers, J., Fantini, S. \& Johansson, P. A review of electrolytes for lithium-sulphur batteries. J. Power Sour. 255, 204-218 (2014).

41. Kang, S. et al. Stretchable lithium-ion battery based on re-entrant microhoneycomb electrodes and cross-linked gel electrolyte. ACS Nano 14, 3660-3668 (2020)

42. Seh, Z. W. et al. Facile synthesis of Li2S-polypyrrole composite structures for high-performance Li2S cathodes. Energy Environ. Sci. 7, 672-676 (2014).

43. Liang, $X$. et al. A highly efficient polysulfide mediator for lithium-sulfur batteries. Nat. Commun. 6, 5682 (2015).

44. Hu, G. et al. 3D graphene-foam-reduced-graphene-oxide hybrid nested hierarchical networks for high-performance Li-S batteries. Adv. Mater. 28, 1603-1609 (2016)

45. Kwon, Y. H. et al. Cable-type flexible lithium ion battery based on hollow multi-helix electrodes. Adv. Mater. 24, 5192-5197 (2012). 